\title{
SSTL IO Standard Based Energy Efficient Digital Clock Design on 28nm FPGA
}

\author{
Shivani Madhok ${ }^{1}$, Navdeep Singh ${ }^{1}$, Furqan Fazili ${ }^{2}$, Sumita Nagah $^{3}$, Sweety Dabas ${ }^{3}$, \\ Ravinder Kaur ${ }^{4}$ and Sweety Dabas ${ }^{5}$ \\ ${ }^{\text {I}}$ Chitkara University, Chandigarh, Punjab \\ ${ }^{2}$ Islamic University of Science \& Technology, Awantipora, India \\ ${ }^{3}$ Gyancity Research Lab, New Delhi, India, \\ ${ }^{4}$ Department of Computer Science, Punjab University, Chandigarh, India \\ ${ }^{5}$ Maharaja Surajmal Institute, Janakpuri, Delhi, India \\ 1shivanimadhok0@gmail.com, 2 furqanfazili@gmail.com, ${ }^{3}$ sumi@gyancity.com, \\ ${ }^{4}$ rkrozy5@gmail.com, ${ }^{5}$ sweety.dabas@gmail.com
}

\begin{abstract}
In this paper we have aimed to have an energy efficient digital clock design. Digital clock is a type of clock that displays time digitally. The code has been implemented in Xilinx ISE Design Suite 14.2 and results were tested on 28nm FPGA platform using Kintex-7 FPGA family using different SSTL IOStandards. Comparison between different SSTL IOStandard has been done to achieve minimum IO power. Via SSTL technology, we achieve green computing with respect to low voltage impedance. In this work we are testing our digital clock design with different SSTL IOStandards such as SSTL15, SSTL18_II, SSTL135, SSTL12, SSTL18_I. In this work we have taken constant value of air flow and heat sink. Airflow has been kept 250 LFM and medium Heat sink. The design consists of five inputs and six outputs. At th end we concluded that there is 24-35\% saving in total power dissipation with $1.2 \mathrm{GHz}$ when compared with $2.2 \mathrm{GHz}$.
\end{abstract}

Keywords: Digital Clock, FPGA, SSTL, IO Standard, Energy Efficient

\section{Introduction}

Digital clock is a type of clock that displays time digitally. Earlier time was indicated by the position of rotating hands [1]. In digital clocks the driven mechanism is not digital [1]. The biggest digital clock is the Lichtzeitpegel ("Light Time Level"), Germany [1]. In this paper, we have attempted to design an energy efficient digital clock and the code has been implemented in Xilinx ISE Design Suite 14.2 and results were tested on $28 \mathrm{~nm}$ FPGA platform using Kintex-7 FPGA family. Nowadays the requirement is for energy efficient devices or the devices that consumes low power to diminish the extra power consumed by the devices. Many attempts have been performed using digital clock. A digital clock design which is based on Nios has also been introduced [2]. Digital designs are suitable for both high-speed clocking and low-voltage applications [3]. Digital methods avoids phase loops or delay line loop which are referred to as analog methods [3]. The objective of this design is to minimize system's dynamic power consumption [4]. Nowadays digital clocks are also present on the rear view mirrors of the vehicles [5]. A general architecture for digital clock for high-speed binary links had also been discussed [6].A paper presents a prototype of a 6- digit digital clock displaying time on a four- digit seven- segment LED module using FPGA (field programmable gate array) [7].Our design of digital clock has been implemented on Xilinx ISE Design Suite 14.2 using different SSTL IOstandards. Comparison between different SSTL IOStandard has been done to achieve minimum IO power. Via SSTL technology, we achieve green computing with 
respect to low voltage impedance [8]. Here we are taking different operating frequencies and testing our digital clock design with different SSTL IOStandards such as SSTL15, SSTL18_II, SSTL135, SSTL12, SSTL18_I.Operating frequencies are listed in Table 3. Airflow and heat sink are main parameters while analyzing the thermal dissipation in the circuit [9]. In this work we have taken constant value of air flow and heat sink. Airflow has been kept 250 LFM and medium Heat sink.Our design consist of five inputs and six 7 bits output as shown in Figure 1. The outsegh1 and outsegh2 are for hours, outsegm1 and outsegm 2 are for hours, outsegs 1 and outsegs 2 are for seconds.

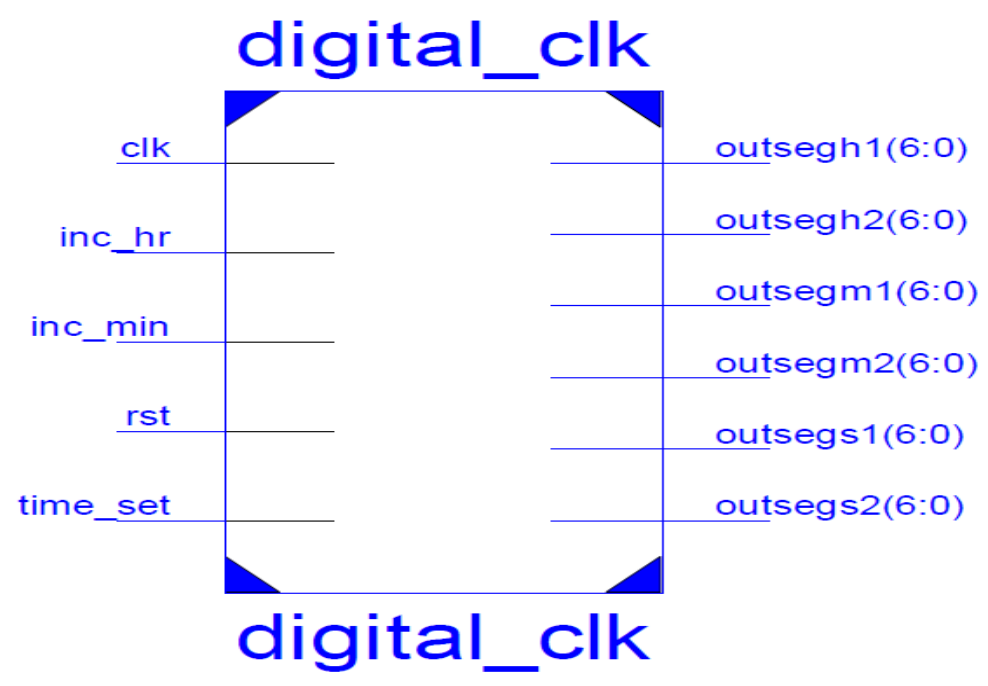

Figure 1. Symbol of Digital Clock

Table 2. Different Parameters in Kintex-7 FPGA

\begin{tabular}{|c|c|}
\hline IO pins & 676 \\
\hline LUT Elements & 101400 \\
\hline Flip Flop & 202800 \\
\hline DSPS & 600 \\
\hline Available IOBS & 400 \\
\hline Gb transceiver & 8 \\
\hline Block RAM & 325 \\
\hline GTXE2 Transceiver & 1.1 \\
\hline PCI Buses & 8 \\
\hline MMCMS & 85 degree Celsius \\
\hline $\begin{array}{c}\text { Min operating } \\
\text { temperature }\end{array}$ & 85 degree Celsius \\
\hline $\begin{array}{c}\text { Reference operating } \\
\text { temperature }\end{array}$ & $0.97 \mathrm{~V}$ \\
\hline $\begin{array}{c}\text { Maximum operating } \\
\text { Temperature }\end{array}$ & $0.97 \mathrm{~V}$ \\
\hline $\begin{array}{c}\text { Minimum operating } \\
\text { voltage }\end{array}$ & $1.03 \mathrm{~V}$ \\
\hline $\begin{array}{c}\text { Reference operating } \\
\text { Voltage }\end{array}$ & $\mathrm{C}$ \\
\hline $\begin{array}{c}\text { Maximum operating } \\
\text { Voltage }\end{array}$ & \\
\hline $\begin{array}{c}\text { Temperature Grade } \\
\text { Letter }\end{array}$ & \\
\hline \multicolumn{2}{|c|}{} \\
\hline
\end{tabular}


Our design "digital clock" is based on the Very High Speed Integrated Circuit Hardware Description Language (VHDL) hardware description language, synthesized by the Xilinx Synthesis Technology (XST) synthesizer [7].This design is based on 28nm FPGA and the code has been tested on Kintex-7 and the device used is XC7K160T, package used is FBG676 and it is working on -3 speed grade. Table 2 shows different parameters in kintex-7 FPGA.

Table 3. Set of Frequencies Taken In Consideration

\begin{tabular}{|c|c|}
\hline Frequency & Mobile set \\
\hline $1400 \mathrm{MHz}$ & Nokia Lumia 710 \\
\hline $1.2 \mathrm{GHz}$ & Samsung Galaxy Core \\
\hline $2100 \mathrm{MHz}$ & I phone6 \\
\hline $1700 \mathrm{MHz}$ & HTC/T \\
\hline $1800 \mathrm{MHz}$ & Micromax X091 \\
\hline $2.2 \mathrm{GHz}$ & Sony Xperia Z1 \\
\hline
\end{tabular}

\section{Power Analysis}

A. Power Analysis for SSTL15 IO STANDARD

Table 4. Power Analysis for SSTL15 IO STANDARD

\begin{tabular}{|c|c|}
\hline FREQUENCY & POWER CONSUMED \\
\hline $1400 \mathrm{MHz}$ & 0.354 \\
\hline $1.2 \mathrm{GHz}$ & 0.323 \\
\hline $2100 \mathrm{MHz}$ & 0.461 \\
\hline $1700 \mathrm{MHz}$ & 0.400 \\
\hline $1800 \mathrm{MHz}$ & 0.416 \\
\hline $2.2 \mathrm{GHz}$ & 0.477 \\
\hline
\end{tabular}

There is $32.28 \%$ saving in total power dissipation with $1.2 \mathrm{GHz}$ when compared with $2.2 \mathrm{GHz}$ as shown in Figure 2 and Table 4.

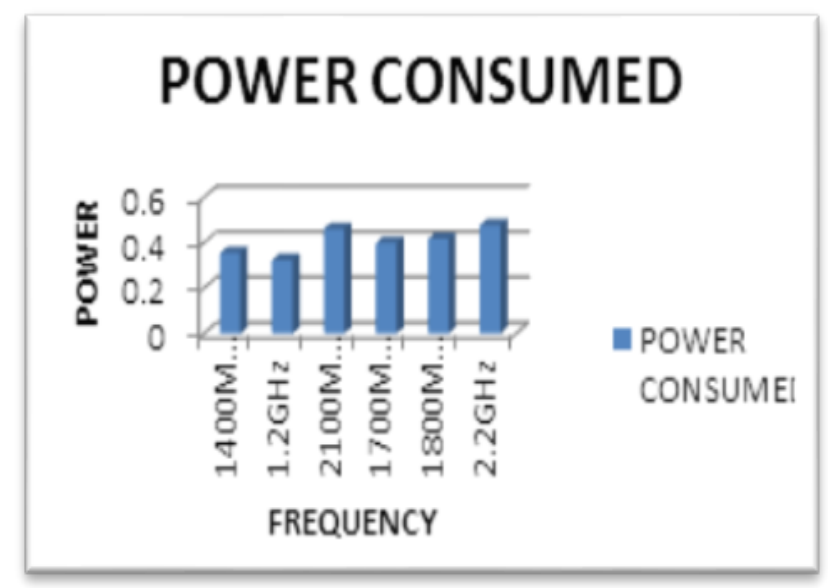

Figure 2. Power Analysis for SSTL15 IO STANDARD 
B. Power Analysis for SSTL18_II IO STANDARD

Table 5. Power Analysis for SSTL18_II IO STANDARD

\begin{tabular}{|c|c|}
\hline FREQUENCY & POWER CONSUMED \\
\hline $1400 \mathrm{MHz}$ & 0.631 \\
\hline $1.2 \mathrm{GHz}$ & 0.573 \\
\hline $2100 \mathrm{MHz}$ & 0.836 \\
\hline $1700 \mathrm{MHz}$ & 0.719 \\
\hline $1800 \mathrm{MHz}$ & 0.749 \\
\hline $2.2 \mathrm{GHz}$ & 0.865 \\
\hline
\end{tabular}

There is $33.75 \%$ saving in total power dissipation with $1.2 \mathrm{GHz}$ when compared with $2.2 \mathrm{GHz}$ as shown in Figure 3 and Table 5.

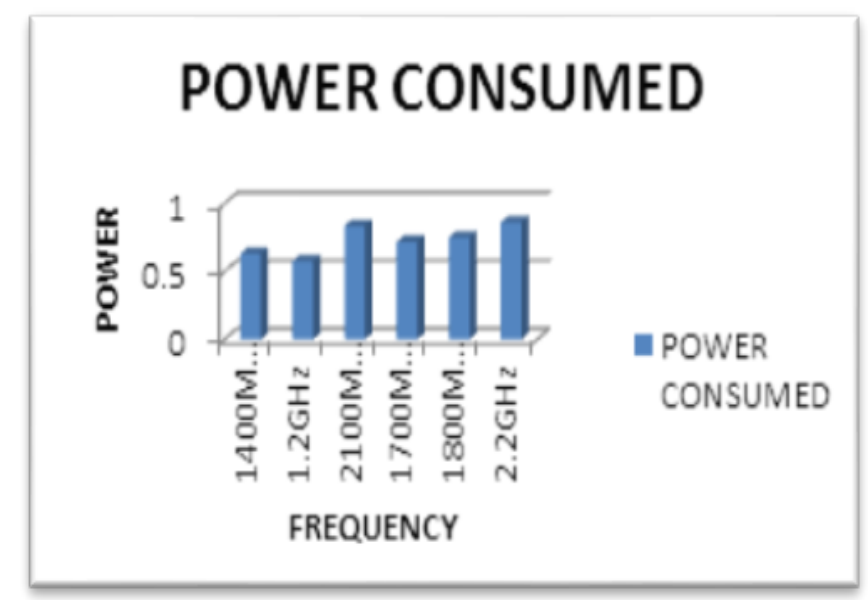

Figure 3. Power Analysis for SSTL18_II IO STANDARD

C. Power Analysis for SSTL135 IO STANDARD

Table 6. Power Analysis for SSTL135 IO STANDARD

\begin{tabular}{|c|c|}
\hline FREQUENCY & POWER CONSUMED \\
\hline $1400 \mathrm{MHz}$ & 0.338 \\
\hline $1.2 \mathrm{GHz}$ & 0.308 \\
\hline $2100 \mathrm{MHz}$ & 0.444 \\
\hline $1700 \mathrm{MHz}$ & 0.523 \\
\hline $1800 \mathrm{MHz}$ & 0.399 \\
\hline $2.2 \mathrm{GHz}$ & 0.460 \\
\hline
\end{tabular}

There is $33.04 \%$ saving in total power dissipation with $1.2 \mathrm{GHz}$ when compared with 2.2GHz as shown in Figure 4 and Table 6. 


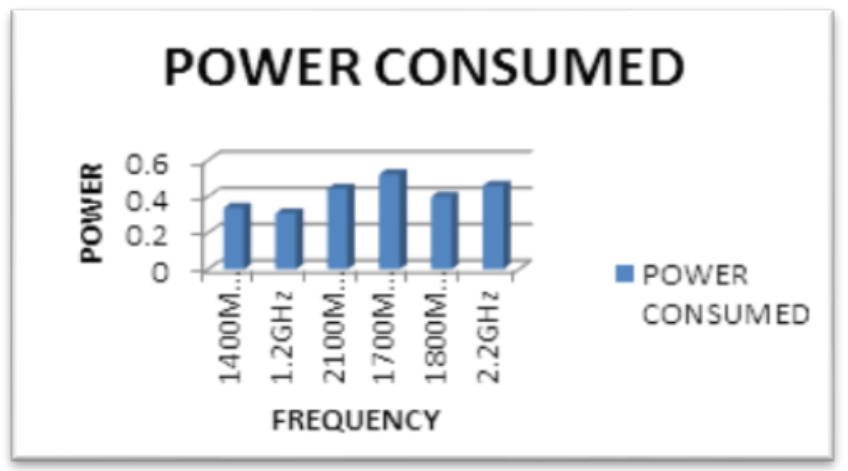

Figure 4. Power Dissipation for SSTL135 IO STANDARD

D. Power Analysis for SSTL12 IO STANDARD

Table 7. Power Analysis for SSTL12 IO STANDARD

\begin{tabular}{|c|c|}
\hline FREQUENCY & POWER CONSUMED \\
\hline $1400 \mathrm{MHz}$ & 0.218 \\
\hline $1.2 \mathrm{GHz}$ & 0.205 \\
\hline $2100 \mathrm{MHz}$ & 0.264 \\
\hline $1700 \mathrm{MHz}$ & 0.237 \\
\hline $1800 \mathrm{MHz}$ & 0.248 \\
\hline $2.2 \mathrm{GHz}$ & 0.270 \\
\hline
\end{tabular}

There is $24.07 \%$ saving in total power dissipation with $1.2 \mathrm{GHz}$ when compared with $2.2 \mathrm{GHz}$ as shown in Figure 5 and Table 7.

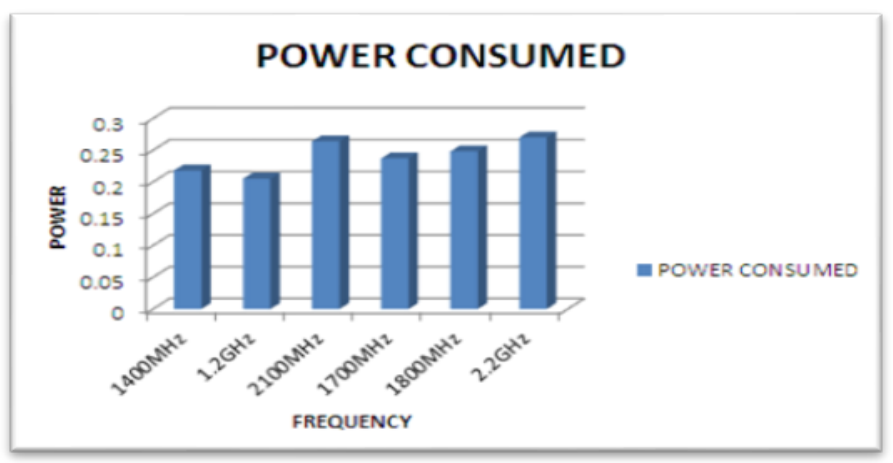

Figure 5. Power Analysis for SSTL12 IO STANDARD

E. Power Analysis for SSTL18_I IO STANDARD

Table 8: Power Analysis for SSTL12 IO STANDARD

\begin{tabular}{|c|c|}
\hline FREQUENCY & POWER CONSUMED \\
\hline $1400 \mathrm{MHz}$ & 0.722 \\
\hline $1.2 \mathrm{GHz}$ & 0.473 \\
\hline $2100 \mathrm{MHz}$ & 0.696 \\
\hline $1700 \mathrm{MHz}$ & 0.598 \\
\hline $1800 \mathrm{MHz}$ & 0.623 \\
\hline $2.2 \mathrm{GHz}$ & 0.722 \\
\hline
\end{tabular}


There is $34.48 \%$ saving in total power dissipation with $1.2 \mathrm{GHz}$ when compared with $2.2 \mathrm{GHz}$ as shown in Figure 6 and Table 8.

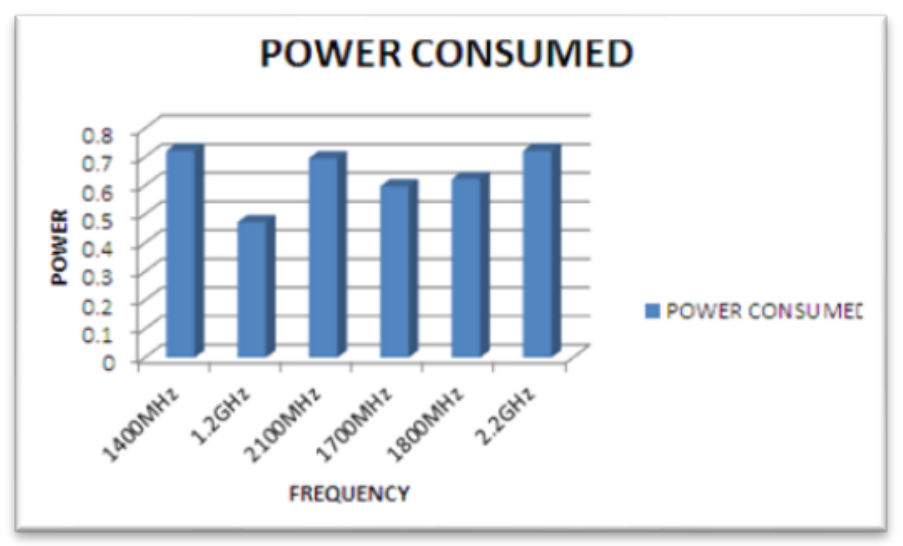

Figure 6. Power Analysis for SSTL12 IO STANDARD

\section{F. Power Analysis for different IO STANDARD with Different Frequencies}

Table 9. Power Analysis For SSTL Family At Different Frequencies

\begin{tabular}{|c|c|c|c|c|c|c|}
\hline $\begin{array}{c}\text { IO } \\
\text { STANDARD }\end{array}$ & $\begin{array}{c}\mathrm{O}^{14} \\
\mathrm{M} \\
\mathrm{Hz}\end{array}$ & $\begin{array}{cc} & 1 . \\
& \\
& G \\
H z\end{array}$ & $\begin{array}{c}{ }^{21} \\
00^{\mathrm{M}} \\
\mathrm{Hz}\end{array}$ & $\begin{array}{c}{ }^{17} \\
00 \\
\mathrm{M} \\
\mathrm{Hz}\end{array}$ & $\begin{array}{c}{ }^{18} \\
00^{\mathrm{M}} \\
\mathrm{Hz}\end{array}$ & $\begin{array}{l}2 . \\
\\
\\
\mathrm{Hz}\end{array}$ \\
\hline d & $\begin{array}{r}0 . \\
354\end{array}$ & $\begin{array}{r}0 . \\
323\end{array}$ & $\begin{array}{r}0 . \\
461\end{array}$ & $\begin{array}{r}0 . \\
400\end{array}$ & $\begin{array}{r}0 . \\
416\end{array}$ & $\begin{array}{r}0 . \\
477\end{array}$ \\
\hline $\begin{array}{l}\text { SSTL18_I } \\
\text { I }\end{array}$ & $\begin{array}{r}0 . \\
631\end{array}$ & $\begin{array}{r}0 . \\
573\end{array}$ & $\begin{array}{r}0 . \\
836\end{array}$ & $\begin{array}{r}0 . \\
719\end{array}$ & $\begin{array}{r}0 . \\
749\end{array}$ & $\begin{array}{r}0 . \\
865\end{array}$ \\
\hline SSTL135 & $\begin{array}{r}0 . \\
338\end{array}$ & $\begin{array}{r}0 . \\
308\end{array}$ & $\begin{array}{r}0 . \\
444\end{array}$ & $\begin{array}{r}0 . \\
523\end{array}$ & $\begin{array}{r}0 . \\
399\end{array}$ & $\begin{array}{r}0 . \\
460\end{array}$ \\
\hline SSTL12 & $\begin{array}{r}0 . \\
218\end{array}$ & $\begin{array}{r}0 . \\
205\end{array}$ & $\begin{array}{r}0 . \\
264\end{array}$ & $\begin{array}{r}0 . \\
237\end{array}$ & $\begin{array}{r}0 . \\
248\end{array}$ & $\begin{array}{r}0 . \\
270\end{array}$ \\
\hline SSTL18_I & $\begin{array}{r}0 . \\
722 \\
\end{array}$ & $\begin{array}{r}0 . \\
473 \\
\end{array}$ & $\begin{array}{r}0 . \\
696 \\
\end{array}$ & $\begin{array}{r}0 . \\
598 \\
\end{array}$ & $\begin{array}{r}0 . \\
623 \\
\end{array}$ & $\begin{array}{r}0 . \\
722 \\
\end{array}$ \\
\hline
\end{tabular}

There is $69.80 \%$ is saving in total power dissipation with SSTL12 when compared with SSTL18_II at $1400 \mathrm{MHz}$.There is $64.22 \%$ saving in total power dissipation with SSTL12 when compared with SSTL18_II at 1.2 GHz.There is $68.42 \%$ saving in total power dissipation with SSTL12 when compared with SSTL18_II at $2100 \mathrm{MHz}$.There is $67.03 \%$ saving in total power dissipation with SSTL12 when compared with SSTL18_II at $1700 \mathrm{MHz}$.There is $66.88 \%$ saving in total power dissipation with SSTL12 when compared with SSTL18_II at $1800 \mathrm{MHz}$.There is $68.78 \%$ saving in total power dissipation with SSTL12 when compared with SSTL18_II at $2.2 \mathrm{GHz}$ as shown in Figure 7 and Table 9. 


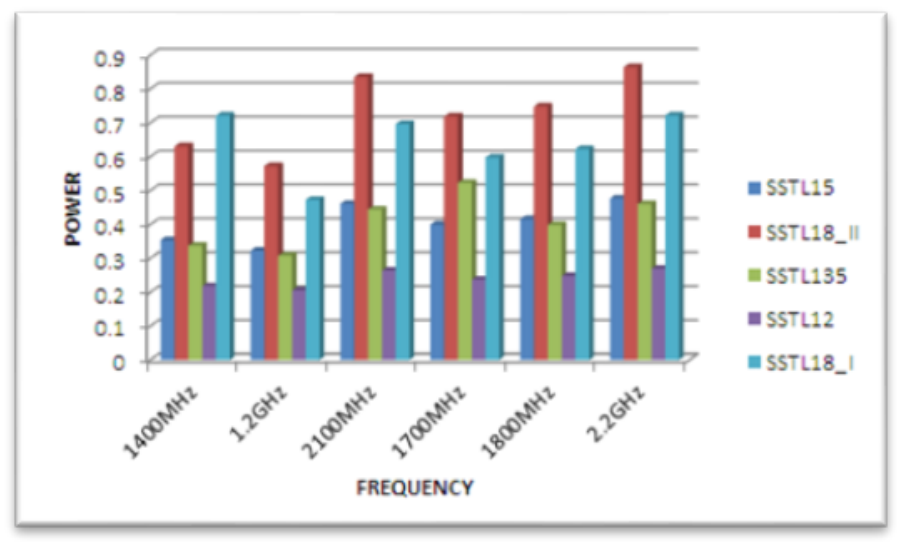

\section{Figure 7. Power Analysis for Sstlfamily at Different Frequencies}

\section{Conclusion}

The design is low power energy efficient and the code has been implemented in Xilinx ISE Design Suite 14.2 and results were tested on $28 \mathrm{~nm}$ FPGA platform using Kintex-7 FPGA family. The digital clock consists of 5 inputs and 6 outputs. Outputs are 7 bits output. The design is tested by varying frequencies at different SSTL IO Standards at constant temperature that is 25 degree Celsius and also keeping air flow constant. We can conclude that there can be $24-35 \%$ saving of total power dissipation by using frequency scaling technique.

\section{Future Scope}

The future scope of "SSTL IO Standard Based Energy Efficient Digital Clock Design on $28 \mathrm{~nm}$ FPGA" is that we can also implement this design on $22 \mathrm{~nm}$ or $18 \mathrm{~nm}$ FPGA. We can also use different FPGA families like automotive Artix7, automotive Coolrunner2, automotive Spartan, automotive Spartan-3A DSP, automotive Spartan 3A, automotive Spartan 3E, automotive Spartan6, Spartan3, Spartan3E. Here, we are using frequency scaling in which we are changing the operating frequency of a device and analyzing its power. We can redesign this Digital Clock Design with other energy efficient technique like capacitance scaling, thermal scaling, clock gating, various design goals, impedance matching with different logic family, and mapping. Analysis has been done with different frequencies like $1400 \mathrm{MHz}, 1.2 \mathrm{GHz}, 2100 \mathrm{MHz}, 1700 \mathrm{MHz}, 1800 \mathrm{MHz}, 2.2 \mathrm{GHz}$. We can use any other frequency range and test our design on that also. The temperature has been kept constant that is 25 degree Celsius so if needed it can also be varied. Air flow can also be varied when required.

\section{References}

[1] http://en.wikipedia.org/wiki/Digital_clock.

[2] X. Guohai, "Digital Clock Design Based on Nios", Signal Processing, 8th International Conference, IEEE, vol. 1, (2006).

[3] P. Nilsson and M. Torkelson, "A monolithic digital clock-generator for on-chip clocking of custom DSP's”, Solid-State Circuits, IEEE Journal, vol. 31, no. 5, (1996), pp. 700-706.

[4] G. E. Téllez, A. Farrahi and M. Sarrafzadeh, "Activity-driven clock design for low power circuits", Proceedings of the 1995 IEEE/ACM international conference on Computer-aided design. IEEE Computer Society, (1995).

[5] R. Pastore, "Combination rear view mirror and digital clock", U. S. Patent No. 4,588,267, (1986) May 13.

[6] J. L. Sonntag and J. Stonick, "A digital clock and data recovery architecture for multi-gigabit/s binary links", Solid-State Circuits, IEEE Journal, vol. 41, no. 8, (2006), pp. 1867-1875.

[7] H. Wan-Fu, "The design of a six-digit digital clock with a four-digit seven-segment display module", Electrical and Control Engineering (ICECE), 2011 International Conference on. IEEE, (2011). 
International Journal of Control and Automation

Vol. 8, No. 6 (2015)

[8] T. Das, "Simulation of SSTL IO standard based power optimized parallel integrator design on FPGA", Robotics and Emerging Allied Technologies in Engineering (iCREATE), 2014 International Conference on. IEEE, (2014).

[9] T. Kumar, "Simulation of voltage based efficient fire sensor on FPGA using SSTL IO standards", Robotics and Emerging Allied Technologies in Engineering (iCREATE), International Conference on. IEEE, (2014). 